\title{
Holographic neutron stars
}

\author{
Jan de Boer, Kyriakos Papadodimas and Erik Verlinde \\ Institute for Theoretical Physics, University of Amsterdam, \\ Valckenierstraat 65, 1018 XE Amsterdam, The Netherlands \\ E-mail: J.deBoer@uva.nl, K.Papadodimas@uva.nl, E.P.Verlinde@uva.nl
}

ABSTRACT: We construct in the context of the AdS/CFT correspondence degenerate composite operators in the conformal field theory that are holographically dual to degenerate stars in anti de Sitter space. We calculate the effect of the gravitational back-reaction using the Tolman-Oppenheimer-Volkoff equations, and determine the "Chandrasekhar limit" beyond which the star undergoes gravitational collapse towards a black hole.

KEYWORDS: Gauge-gravity correspondence, AdS-CFT Correspondence

ARXIV EPRINT: 0907.2695 


\section{Contents}

1 Introduction 1

2 Degenerate conformal operators $\quad 1$

3 Hydrodynamic description $\quad 4$

$\begin{array}{lll}4 & \text { Including self gravity } & 6\end{array}$

$\begin{array}{lll}5 & \text { Results and discussion } & 7\end{array}$

\section{Introduction}

A lot of progress has been made in string theory in understanding the most extreme form of matter, namely black holes. String theory should also be able to describe less extreme forms of matter, such as the degenerate matter that makes up neutron stars. A neutron star is able to withstand the force of gravity as long as the total mass stays below a critical value referred to as the Oppenheimer-Volkoff limit [1,2]. This critical mass is determined by solving the Einstein equations for spherically symmetric pressure and energy density distributions, also known as the Tolman-Oppenheimer-Volkoff equations.

It would be especially interesting to study the collapse of a neutron star towards a black hole in a context with a holographic description. In this letter we take a first step in this direction by studying degenerate stars in Anti-de Sitter space, and constructing the corresponding states within the conformal field theory. One of our aims is to determine the OV limit for these degenerate stars and to give its holographic interpretation in terms of the CFT. Previous studies of stars in AdS are found in [3-6].

We begin in section II by describing degenerate composite operators in the CFT constructed out of many fermionic single trace primary fields. In section III we show that these operators correspond to a degenerate Fermi gas in AdS space, and we present a hydrodynamic description. We subsequently define a double scaling limit, and argue that in this limit the mass and Fermi energy receive corrections due to self gravity only. The TOV equations in AdS space and their analytic properties are described in section IV. In section $\mathrm{V}$ we present our numerical results, compare with the boundary calculations and conclude with remarks on the interpretation.

\section{Degenerate conformal operators}

We consider a CFT defined on the cylinder $R \times S^{d-1}$ with metric

$$
d s^{2}=-d t^{2}+\ell^{2} d \Omega_{d-1}^{2},
$$


where $d \Omega_{d-1}^{2}$ represents the standard metric on $S^{d-1}$. The cylinder is identified with the boundary of global anti de Sitter space $A d S_{d+1}$ with curvature radius $\ell$. To make use of the operator state correspondence, we analytically continue to euclidean time and conformally map the cylinder on the euclidean plane $R^{d}$ by

$$
t \rightarrow i \ell \log |x| .
$$

The angular coordinates $\Omega$ are identified in the obvious way. States in the CFT correspond to operators via

$$
\lim _{|x| \rightarrow 0} \Phi(x)|\operatorname{vac}\rangle=|\Phi\rangle .
$$

We now assume that the CFT contains bosonic as well as fermionic fields, and has a large $c$ limit in which one can distinguish single and multi trace operators. ${ }^{1}$

Our aim is to find a degenerate state made out of fermions. So let us choose a fermionic single trace primary operator $\Psi$ with conformal dimension $\Delta_{0}$. $\Psi$ is assumed to be complex so that it differs from its conjugate $\bar{\Psi}$. We first consider the limit $c \rightarrow \infty$, while keeping all other quantities fixed. In this limit single trace primaries behave as "free" fields, since three and higher point functions are suppressed by powers of $1 / \sqrt{c}$, see footnote [5]. By spin-statistics, fermionic operators anti-commute

$$
\Psi(x) \Psi(y)=-\Psi(y) \Psi(x),
$$

and hence they generate a free fermionic Fock space. The lowest dimension composites of two fields $\Psi$ are given by $\Psi \partial_{i} \Psi$, where $\partial_{i}$ denotes the derivative with respect to $x_{i}$. We continue with a third fermionic operator, a fourth, up to a very large number, each time making sure that the next operator is anti-symmetrized with respect to the previous ones by acting with one, two, three derivatives, etc. For a degenerate state one uses the smallest number of derivatives necessary to avoid that any given combination occurs twice. For simplicity we ignore spinor indices. In this way we arrive at the following composite operator

$$
\begin{gathered}
\boldsymbol{\Phi}=\Psi \prod_{i} \partial_{i} \Psi \prod_{\{i, j\}} \partial_{i} \partial_{j} \Psi \prod_{\{i, j, k\}} \partial_{i} \partial_{j} \partial_{k} \Psi \ldots \\
\ldots \ldots \prod_{\left\{i_{1}, i_{2}, \ldots i_{n_{F}}\right\}} \partial_{i_{1}} \partial_{i_{2}} \ldots \partial_{i_{n_{F}}} \Psi
\end{gathered}
$$

The fermionic primaries are arranged in "shells" labeled by the number of derivatives, where in each shell the product is taken over all possible $n$-tuples of the derivatives $\partial_{i}$. The number of derivatives in the last shell is denoted by $n_{F}$. We consider the case with a completely filled last shell, so that we have a rotationally symmetric operator. The total number of fields contained in $\Phi$ may then be expressed in terms of binomial coefficients as

$$
N=\sum_{n=0}^{n_{F}}\left(\begin{array}{c}
n+d-1 \\
d-1
\end{array}\right) .
$$

\footnotetext{
${ }^{1}$ Throughout this paper we use $c$ instead of the rank $N$. For $\operatorname{SU}(N) \mathcal{N}=4 \mathrm{SYM}$ the relation is: $N^{2}-1=\pi^{4} c / 10$.
} 
Since each derivative carries conformal dimension one, each operator in the $n$-th shell has dimension $n+\Delta_{0}$. Hence the total conformal dimension of $\boldsymbol{\Phi}$ equals

$$
\Delta=\sum_{n=0}^{n_{F}}\left(n+\Delta_{0}\right)\left(\begin{array}{c}
n+d-1 \\
d-1
\end{array}\right) .
$$

The primary operator $\Psi$ corresponds to a fermionic field $\psi$ in the bulk. The number of derivatives acting on $\Psi$ is directly related to the quantum number $n$ that determines the energy of the partial waves of $\psi$, see e.g. [7]. The operator $\Phi$ represents a degenerate many particle state, whose complete wave function is given by the Slater determinant of all partial waves with $n \leq n_{F}$.

Let us now introduce the total mass $M$, the fermion mass $m$, and the Fermi energy $\epsilon_{F}$ via

$$
m=\Delta_{0} / \ell, \quad M=\Delta / \ell, \quad \epsilon_{F}=\left(n_{F}+\Delta_{0}\right) / \ell .
$$

We are interested in the limit of large $n_{F}$ (and large $\Delta_{0}$ ), so it is more convenient to rewrite the sums in the expressions (2.5) and (2.6) as integrals. This gives

$$
N\left(\epsilon_{F}\right)=\int_{m}^{\epsilon_{F}} d \epsilon g(\epsilon), \quad M\left(\epsilon_{F}\right)=\int_{m}^{\epsilon_{F}} d \epsilon \epsilon g(\epsilon),
$$

where the density of states $g(\epsilon)$ is given by

$$
g(\epsilon)=\frac{\ell^{d}}{(d-1) !}(\epsilon-m)^{d-1}
$$

We would like to emphasize that our discussion so far has been exact for all values of the coupling of the gauge theory, as long as $c \rightarrow \infty$. We have assumed that the basic building block $\Psi$ of our operators has conformal dimension $\Delta_{0}$. For our purposes we do not care whether this $\Delta_{0}$ is the same as the conformal dimension of $\Psi$ at weak coupling. Instead for us $\Delta_{0}$ can be considered as an input parameter which is determined by the (possibly strongly coupled) dynamics. The operator $\Psi$ is dual to the bulk fermionic field of mass $m$ via the standard AdS/CFT correspondence, and therefore at strong coupling we only care about the relation between the mass $m$ and the dimension $\Delta_{0}$, which is fixed by the AdS/CFT dictionary. For large values of $\Delta_{0}$ this relation simply becomes $m=\Delta_{0} / \ell$. But even if we wanted to find an operator whose dimension $\Delta_{0}$ is exactly known at strong 't Hooft coupling we could have taken $\Psi$ to be a fermionic descendant of a chiral primary in a supersymmetric theory. ${ }^{2}$ Generally there will be many candidate fermionic operators that can be used as the basic fermionic operator $\Psi$. The qualitative aspects of our analysis are not sensitive to details of these operators, so any of them could be used. Different choices of the fermionic operator on the boundary correspond to degenerate states in the bulk made out of fermions of different "flavor".

On the other hand the operator $\boldsymbol{\Phi}$ defined in (2.4) is definitely not supersymmetric, even if $\Psi$ is. The derivatives increase the conformal dimension without adding R-charge,

\footnotetext{
${ }^{2}$ For example in the $\mathcal{N}=4 \mathrm{SYM}$ we could have taken $\Psi$ to be a fermionic descendant of a chiral primary i.e. $\Psi=\left[Q, \operatorname{Tr} Z^{n}\right]$.
} 
hence the operator $\boldsymbol{\Phi}$ does not saturate the BPS bound. Nevertheless, the expression (2.6) is still reliable due to the 't Hooft factorization at large $c$. It is well known that the conformal dimensions of multi-trace operators are equal to the sum of the individual conformal dimensions up to $1 / c$ corrections. This statement is based on the planar expansion and does not depend on the value of the 't Hooft coupling. Hence in the limit $c \rightarrow \infty$ our formula (2.6) is exact. The 't Hooft factorization also guarantees that at large $c$ we do not have mixing of the operator $\boldsymbol{\Phi}$ with other operators with the same quantum numbers. Hence the operator $\boldsymbol{\Phi}$ is the unique operator with smallest conformal weight (at infinite c) with given fermion number ${ }^{3}$ for $\Psi$. These statements are reliable if we take $c \rightarrow \infty$ keeping other quantities (like $\Delta_{0}$ and $n_{f}$ ) fixed.

In the following we define a double scaling limit that takes $n_{F}$ and $c$ simultaneously to infinity, while keeping the ratio $\Delta / c$ fixed. In this limit the 't Hooft suppression partially breaks down, and therefore there will be corrections to the conformal dimension $\Delta$ and density of states $g(\epsilon)$ defined in (2.8). The main correction to $\Delta$ turns out to come from $t$-channel exchange of the stress tensor, and can be estimated to be of the order of $\Delta^{2} / c$, see [8]. This corresponds to the inclusion of self gravity in the bulk.

When operator mixing sets in, the state $|\Phi\rangle$ has to be defined a little more carefully. Suppose we have a state $|\Phi\rangle_{N}$ composed out of $N$ operators $\Psi$. We then define

$$
\Psi(x)|\Phi\rangle_{N} \sim x^{\left(m-\epsilon_{F}\right) \ell}|\Phi\rangle_{N+1},
$$

where one chooses $\epsilon_{F}$ so that one picks up (one of) the lowest order term(s) on the right hand side. For $\Delta / c \rightarrow 0$ this should lead to the operator (2.4). This procedure is well defined as long as the density of states $g\left(\epsilon_{F}\right)$ remains finite. When $g\left(\epsilon_{F}\right)$ diverges the definition of $\Phi$ becomes obscure. We will see this is precisely what happens at the OppenheimerVolkoff limit.

\section{Hydrodynamic description}

We return to the case where first $c \rightarrow \infty$, and then take a subsequent limit in which the number of particles per AdS volume goes to infinity while keeping the ratio $\epsilon_{F} / m$ fixed. In this hydrodynamic limit the particles form an ideal degenerate Fermi gas. The number of particles within one AdS radius is very large, and therefore the energy density $\rho$ and pressure $p$ obey the same equation of state as in flat space. Specifically, the particle number density $\mathbf{n}$ equals the volume of the Fermi sphere

$$
\mathbf{n}=\frac{V_{d-1}}{d(2 \pi)^{d}} k_{F}^{d}
$$

where $V_{d-1}$ denotes the area of $S^{d-1}$. The Fermi momentum $k_{F}$ is related to the chemical potential $\mu$ via

$$
\mu=\sqrt{k_{F}^{2}+m^{2}} .
$$

\footnotetext{
${ }^{3}$ The notion of a fermion number makes sense at infinite $c$ since all interactions are suppressed due to factorization.
} 
The energy density and pressure are given by the "textbook" expressions

$$
\begin{aligned}
& \rho=\frac{V_{d-1}}{(2 \pi)^{d}} \int_{0}^{k_{F}} d k k^{d-1} \sqrt{k^{2}+m^{2}}, \\
& p=\frac{V_{d-1}}{d(2 \pi)^{d}} \int_{0}^{k_{F}} d k \frac{k^{d+1}}{\sqrt{k^{2}+m^{2}}},
\end{aligned}
$$

and obey the standard thermodynamic relations

$$
d \rho=\mu d \mathbf{n}, \quad \rho+p=\mu \mathbf{n}
$$

We want to determine the density and pressure profile corresponding to the operator $\Phi$. We will do so first for the case of pure AdS, but in the following section we generalize these results to include self-gravity. Anticipating these generalizations, let us write the metric in the form

$$
d s^{2}=-A(r)^{2} d t^{2}+B(r)^{2} d r^{2}+r^{2} d \Omega_{d-1}^{2} .
$$

The stress energy tensor of the Fermi gas is given by the standard hydrodynamic expression

$$
T_{\mu \nu}=(\rho+p) u_{\mu} u_{\nu}+p g_{\mu \nu}
$$

where $u_{\mu}$ is a static velocity field: $u_{\mu} d x^{\mu}=A(r) d t$. The radial profiles of $p$ and $\rho$ are determined by imposing stress energy conservation. This leads to the condition

$$
\frac{d p}{d r}+\frac{1}{A} \frac{d A}{d r}(\rho+p)=0
$$

This equation is surprisingly easy to solve. By making use of the identities (3.4) one easily verifies that (3.7) is satisfied when the chemical potential obeys

$$
\mu(r)=\frac{\epsilon_{F}}{A(r)},
$$

where at this stage $\epsilon_{F}$ is an arbitrary constant. We conclude that the radial dependence of the chemical potential is simply due to the gravitational redshift. We now observe that the constant $\epsilon_{F}$ equals the chemical potential defined with respect to the time $t$. This means that it can be identified with the Fermi energy $\epsilon_{F}$ of the CFT.

When the chemical potential $\mu$ is equal to the fermion mass $m$, the pressure and energy density drop to zero. From (3.8) we find that this happens at $r=R$ for which

$$
A(R)=\frac{\epsilon_{F}}{m}
$$

This defines the edge of the star. Note that the edge is only present when the fermions have a finite mass.

Up to this point our discussion holds for any metric of the form (3.5). We now specialize to the case of pure AdS space, so that $A^{2}=1 / B^{2}=1+r^{2} / \ell^{2}$. One then finds that the radius $R$ of a free Fermi gas in AdS is determined by the Fermi momentum at the center: $R / \ell=k_{F}(0) / m$. 
As a non-trivial check on our hydrodynamic description one can verify that the particle number and mass agree with the boundary calculations. The total particle number is given by the integral

$$
N=V_{d-1} \int_{0}^{R} d r r^{d-1} B(r) \mathbf{n}(r)
$$

The factor $B(r)$ comes from the volume form on the spatial section. Similarly, one obtains the mass $M$ by integrating the energy density. Because $M$ is defined with respect to the time coordinate $t$ one has to include a redshift factor $A(r)$. For AdS this cancels the measure factor $B(r)$, and hence

$$
M=V_{d-1} \int_{0}^{R} d r r^{d-1} \rho(r)
$$

In fact, this expression is equivalent to the ADM mass, and also holds when self gravity is included. To calculate $N$ and $M$ one has to insert the equations (3.1) and (3.3) for the particle and energy density and include the redshift effect (3.8) into the chemical potential. The resulting integrals can be performed analytically and (perhaps not) surprisingly precisely reproduce the results (2.8) and (2.9).

\section{Including self gravity}

Having established that for infinite $c$ the operator $\Phi$ is represented by a free degenerate Fermi gas, we now proceed to the double scaling limit $n_{F} \rightarrow \infty$ and $c \rightarrow \infty$ with fixed values for

$$
\frac{n_{F}}{\Delta_{0}}=\frac{\epsilon_{F}}{m}-1, \quad \frac{\Delta}{c} \sim \frac{G M}{\ell^{d-2}}
$$

One may think this leads not only to gravitational interactions, but also possibly makes the fermions unstable. However, the 't Hooft suppression still seems to be sufficiently active to ensure that the Fermi gas has an infinite life time. The possible depletion of the particle number density is controlled by the Boltzman equation

$$
\frac{d \mathbf{n}}{d t} \sim \frac{k_{F}}{m} \mathbf{n}^{2} \sigma
$$

where $\sigma$ represents the total cross section associated with the decay. In the double scaling limit $\mathbf{n}$ grows like $c^{d /(d+1)}$, while $\sigma$ contains at least one factor of $c^{-1}$ due to 't Hooft suppression. So, unless $\sigma$ contains other factors of $c$, the life time of the fermions is estimated to be of the order of $c^{1 /(d+1)}$, hence is infinite for $c \rightarrow \infty$. The decay rates are possibly enhanced by energy or phase space factors. Various checks, involving dimensional arguments, softness of string amplitudes and the optical theorem, indicate that these effects do not ruin our argument. For a more detailed discussion we refer to [8].

So let us now assume that gravity is the only force. ${ }^{4}$ Except for including gravity, the fermionic matter will be treated exactly as in the previous section. We parametrize the

\footnotetext{
${ }^{4}$ For chiral primaries there is also a gauge force due to the $R$-charge. One finds that the results are qualitatively the same as presented here: gravity eventually wins [8].
} 
functions $A(r)$ and $B(r)$ in terms of two new functions $M(r)$ and $\chi(r)$ as

$$
\begin{aligned}
& A^{2}(r)=e^{2 \chi(r)}\left(1-\frac{C_{d} M(r)}{r^{d-2}}+\frac{r^{2}}{\ell^{2}}\right), \\
& B^{2}(r)=\left(1-\frac{C_{d} M(r)}{r^{d-2}}+\frac{r^{2}}{\ell^{2}}\right)^{-1},
\end{aligned}
$$

where Newton's constant is contained in the coefficient

$$
C_{d}=\frac{16 \pi G}{(d-1) V_{d-1}} .
$$

In terms of $M(r)$ and $\chi(r)$ the Einstein equations read

$$
\begin{aligned}
M^{\prime}(r) & =V_{d-1} \rho(r) r^{d-1} \\
\chi^{\prime}(r) & =V_{d-1} \frac{C_{d}}{2}(\rho(r)+p(r)) r B^{2}(r) .
\end{aligned}
$$

Together with (3.7) these constitute the TOV equations. We choose as boundary condition $M(0)=0$, so that $M(r)$ represents the contribution to the mass from the energy density inside a ball of radius $r$, and the total mass is equal to $M(R)$. By Birkhoff's theorem the metric outside the star is given by AdS-Schwarzschild. Hence,

$$
\chi(r)=0, \quad M(r)=M, \quad \text { for } r \geq R .
$$

From (3.9) it follows that the radius $R$ is determined by

$$
1-\frac{C_{d} M}{R^{d-2}}+\frac{R^{2}}{\ell^{2}}=\left(\frac{\epsilon_{F}}{m}\right)^{2} .
$$

We want to use the TOV equations to calculate the corrections to the mass, the particle number and the density of states defined through the relations (2.8). In fact, to show that the relations (2.8) are still valid one needs to use the Einstein equations! One has

$$
\begin{aligned}
\epsilon_{F} \frac{d N}{d \epsilon_{F}} & =V_{d-1} \int_{0}^{R} d r r^{d-1}\left(B \epsilon_{F} \frac{d \mathbf{n}}{d \epsilon_{F}}+\frac{d B}{d \epsilon_{F}} \epsilon_{F} \mathbf{n}\right) \\
& =V_{d-1} \int_{0}^{R} d r r^{d-1} e^{\chi}\left(\frac{d \rho}{d \epsilon_{F}}+\frac{d B}{d \epsilon_{F}} \frac{\rho+p}{B}\right) \\
& =\int_{0}^{R} d r e^{\chi}\left(\frac{d M^{\prime}}{d \epsilon_{F}}+\frac{d M}{d \epsilon_{F}} \chi^{\prime}\right)=\frac{d M}{d \epsilon_{F}} .
\end{aligned}
$$

Here we used the identity (3.4), the relation (3.8), the TOV equations (4.3), and the definitions of $A$ and $B$ in terms of the functions $M$ and $\chi$.

\section{Results and discussion}

The TOV equations can be integrated numerically. We give the results for $d=4$, so for gravity in $A d S_{5}$. Other cases are qualitatively similar. Just as in [1-6] we find that the mass $M$ reaches a maximum as a function of the chemical potential at the center, see figure 


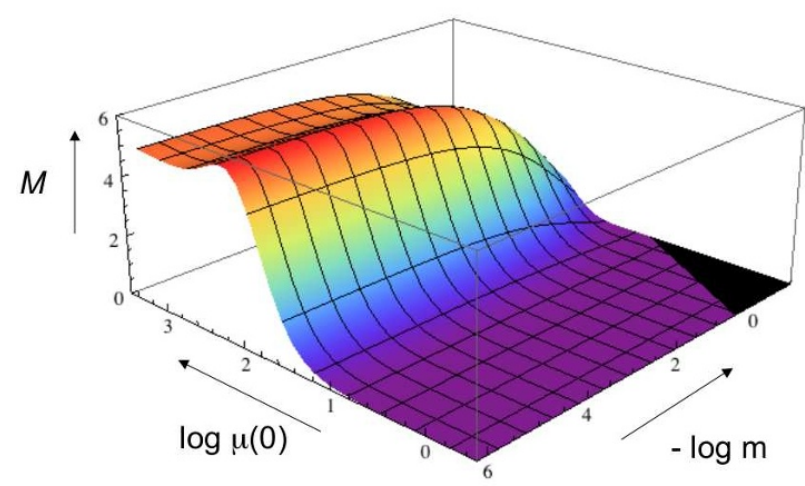

Figure 1. $M$ as a function of $\log \mu(0)$ plotted against $\log m$ for $A d S_{5}$. The black corner is the "forbidden" region $\mu(0)<m$.

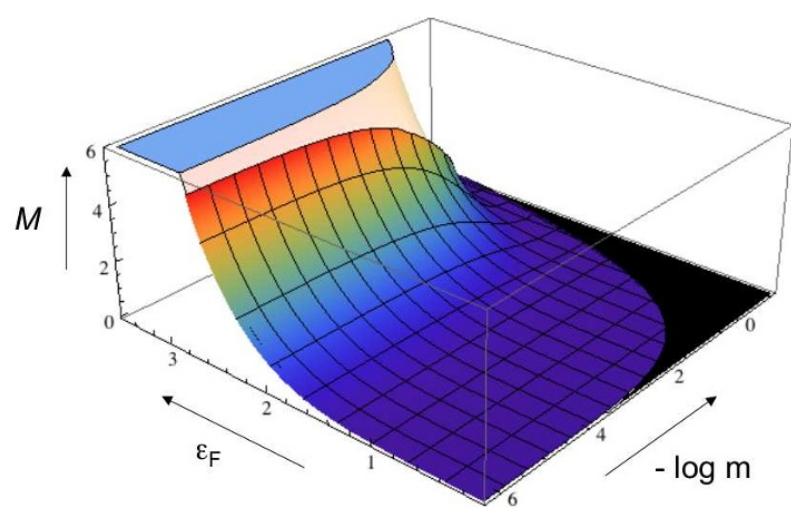

Figure 2. $M\left(\epsilon_{F}\right)$ plotted against $\log m$. The underlying pink graph is the result without gravity. The units in all figures are $M=\Delta / c, \epsilon_{F}=\left(n_{F}+\Delta_{0}\right) / c^{1 /(d+1)}$ and $m=\Delta_{0} / c^{1 /(d+1)}$.

1. Beyond this critical value the solution becomes unphysical. Similar plots are found for $N$ and $\epsilon_{F}$ as a function of $\mu(0)$. From a holographic viewpoint it is more natural to regard $M$ as a function of the Fermi energy $\epsilon_{F}$. This leads to the graph shown in figure 2. The upper edge of the colored part is the OV limit, while the underlying graph is the un-backreacted result (2.8) and (2.9). The effect of self gravity on the value of $M$ is surprisingly small. The same holds for $N$. Yet on the density of states defined as $g(\epsilon)=d N / d \epsilon$ the effect is significant: it diverges at the $\mathrm{OV}$ limit as shown in figure 3. This is a clear sign of an instability.

Of course, one would like to derive the corrections to the mass from the boundary perspective. Qualitatively it is clear that these corrections are due to operator mixing. More importantly, we like to find the meaning of the OV limit in the CFT. In the bulk the star becomes unstable under a radial density perturbation. It would be interesting to interpret this mode in the CFT. Beyond the OV limit the star presumably collapses and forms a black hole. In the CFT this means that the operator $\Phi$ starts to mix with generic states, and thermalizes. This suggests that the collapse is associated with a phase transition that turns a high density (baryonic) state into a thermal state (quark gluon plasma). Clearly these questions and the stability issue need further study. 


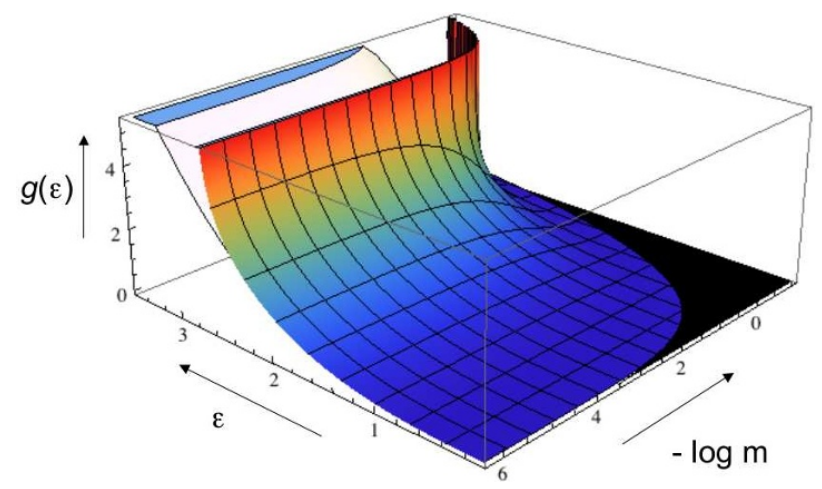

Figure 3. The density of states $g(\epsilon)$ plotted against $\log m$. The underlying graph is the result $(2.9)$ without back-reaction. Here and in figure 2 the black region corresponds to $\epsilon_{F}<m$.

\section{Acknowledgments}

We would like to thank O. Aharony, S. Hellerman, G. Horowitz, V. Hubeny, E. Kiritsis, J. Maldacena, P. McFadden, S. Minwalla, B. Nienhuis, H. Ooguri, M. Rangamani, L. Rastelli, K. Schoutens, M. Shigemori, A. Strominger, M. Taylor, S. Trivedi, and H. Verlinde for discussions. This work was partly supported by the Foundation of Fundamental Research on Matter (FOM).

Open Access. This article is distributed under the terms of the Creative Commons Attribution Noncommercial License which permits any noncommercial use, distribution, and reproduction in any medium, provided the original author(s) and source are credited.

\section{References}

[1] J.R. Oppenheimer and G.M. Volkoff, On massive neutron cores, Phys. Rev. 55 (1939) 374 [SPIRES].

[2] R.C. Tolman, Static solutions of Einstein's field equations for spheres of fluid, Phys. Rev. 55 (1939) 364 [SPIRES].

[3] D.N. Page and K.C. Phillips, Selfgravitating radiation in anti-De Sitter space, Gen. Rel. Grav. 17 (1985) 1029 [SPIRES].

[4] V.E. Hubeny, H. Liu and M. Rangamani, Bulk-cone singularities 83 signatures of horizon formation in AdS/CFT, JHEP 01 (2007) 009 [hep-th/0610041] [SPIRES].

[5] V. Vaganov, Self-gravitating radiation in $A d S(d)$, arXiv:0707.0864 [SPIRES].

[6] J. Hammersley, A critical dimension for the stability of perfect fluid spheres of radiation, Class. Quant. Grav. 25 (2008) 205010 [SPIRES].

[7] O. Aharony, S.S. Gubser, J.M. Maldacena, H. Ooguri and Y. Oz, Large-N field theories, string theory and gravity, Phys. Rept. 323 (2000) 183 [hep-th/9905111] [SPIRES].

[8] X. Arsiwalla, J. de Boer, K. Papadodimas and E.P. Verlinde, Degenerate stars and gravitational collapse in $A d S / C F T$, to appear. 